\title{
Fusing multiple sources of context data of the same context type
}

\author{
Markus C. Huebscher, Julie A. McCann, Naranker Dulay \\ Department of Computing \\ Imperial College London \\ \{mch1,jamm,nd\}@doc.ic.ac.uk
}

\begin{abstract}
Smart homes are expected to have sensors that monitor the home and its inhabitants. Sensors types include temperature sensors, pressure sensors in the floor, or even accelerometers worn by the inhabitants. Data from these sensors can be used to infer higherlevel context, such as the current activity of the inhabitants. We look at the case where there are multiple providers of the same type of context in the home, and describe a probabilistic mechanism for combining these multiple context outputs into a single output using the advertised probability of correctness of each provider. We show that the resulting context value has a higher quality (specifically a higher probability of correctness) than selecting and using any of the available providers on their own.
\end{abstract}

\section{Introduction}

Future smart homes are expected to be filled with sensors for the purpose of sensing physical properties of the home and its inhabitants. Sensors may be builtin to the walls or floor, such as in MIT's PlaceLab [8] and Georgia Tech's Aware Home [6], and might also consist of video cameras, as in Microsoft Research's EasyLiving project [1]. From the sensed data, the objective is often to infer the current activity of the inhabitants and their well-being $[9,14]$, which is particularly important for monitoring elderly or convalescent people, who are thereby able to spend more time living autonomously in their home instead of being taken to a nursing home or hospital.

Our work starts with the assumption that in smart homes there will be multiple ways of determining a type of contextual information, such as a person's activity, using different sets of sensors or inference techniques. Based on this assumption, we present a technique for combining context data of the same type from different sources into a single output, such that all inputs and their currently advertised probability of correctness are taken into account. We show that the resulting output context is better than the output of any of the available providers taken on their own.

\subsection{Related work}

There has been considerable work in pervasive computing middleware frameworks on selecting the most suited available service to accomplish the tasks of a context-aware application.

For instance, in Gaia [11] applications can query the middleware layer for context information using first-order logic and boolean algebra. The Solar System [2], the Strathclyde Context Infrastructure [15] and iQueue [3] also use essentially boolean predicates to determine if an available provider of context fits the requirements of the application, by comparing the values of the properties of provider with the values specified by the application.

Instead, previous work of ours [5] uses applicationdependent utility functions to map the attributes of a provider to a numerical satisfaction value, so that the provider with highest utility is chosen as the best alternative for the application. Also Gaia was recently been extended with a high-level programming model [10] that allows selection of a service based on the ranking of certain quantitative properties, e.g. ranking by ascending location distance from the service.

Our work complements service selection in pervasive middleware frameworks when the most important attribute is the precision of the context data received by the applications. Indeed, for certain types of context, such the activity of a person, the main factor for selecting a particular provider is its precision. We show that to this end, in order to deliver to applications context data with the highest possible precision, it makes sense for these types of context information to combine the output of multiple providers of the same type of context into a single output, rather than selecting and using only one of the available providers. Further, context is often location-dependent, or more generally dependant on some other type of context. We also show how taking this dependency into account can further improve the quality of the resulting context data.

\subsection{Scenario and approach}

We will describe and show the usefulness of our technique by working on the example of the context 


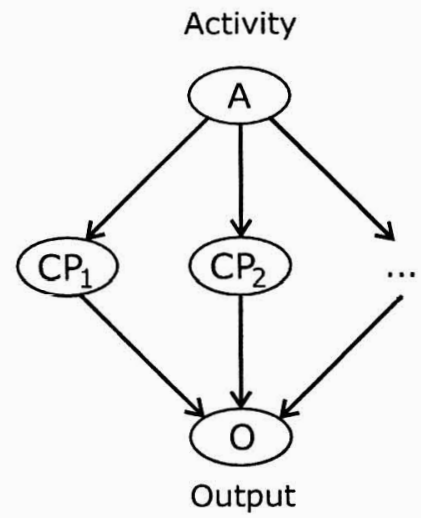
Figure 1. Bayesian network for combin-
ing output of multiple CPs.

type "activity" in a smart home, using for simplicity the values standing, sitting and lying. The goal is to take as input the context of all available providers in the smart home of this type of context and output a final context value that takes into account each input, including their advertised probability of correctness poc, such that the resulting output is better than any of the available inputs taken on their own. Our approach is generally applicable to any context with a finite set of discrete context values. While it is potentially also applicable to context types with continuous output, it is more difficult to describe a generic approach that works well with continuous context types.

As we consider in the decision making process probabilities in the input (poc), we choose to use statistical inference techniques to solve this task, and therefore model the relationship between the actual activity, the output of the context providers detecting the activity and the final resulting output as a Bayesian network.

\section{Bayesian network}

Let us start by describing the Bayesian network (BN) on we which our strategy for combining context inputs is based. Figure 1 shows our BN instance for the context type "activity", although it will have the same structure for most context types. A BN is a directed acyclic graph, where the nodes of the graph represent random variables, and the directed links indicate that the parent node has a direct influence on the child node [12]. Thus, in Figure 1 the activity being monitored (node $A$ ) has a direct influence on the context providers (CPs) that are trying to determine that activity through their sensors; and the outputs of the CPs (nodes $\mathrm{CP}_{1}, \mathrm{CP}_{2}, \ldots$ ) have a direct influence on the final output (node $\mathrm{O}$ ), as it takes into account the output of each $\mathrm{CP}$ and their advertised probability of correctness (poc).

Broadly, any BN is used in the following way. First, conditional probabilities are defined for each random variable in the $\mathrm{BN}$, based either on training data or some knowledge of the problem domain. In our case, this means defining the probabilities $P(A), P\left(C P_{1} \mid A\right)$, $P\left(C P_{2} \mid A\right)$, etc. and $P\left(O \mid C P_{1}, C P_{2}, \ldots\right)$. Once this is done, the network describes the full joint probability distribution of the domain, i.e. it is possible to determine the probability of each outcome of any of the random variables in the $\mathrm{BN}$. Ultimately of interest to us is $P\left(A \mid O, C P_{1}, \ldots, C P_{n}\right)$, i.e. how likely each value for the actual activity is, given the final output and the output of each CP. In fact, we compute this probability for every possible value and then choose as the final output the value that is most likely.

Using the BN in Figure 1 and Bayes' product rule

$$
P\left(x_{1}, \ldots, x_{n}\right)=\prod_{i=1}^{n} P\left(x_{i} \mid \operatorname{parents}\left(X_{i}\right)\right)
$$

where parents $\left(X_{i}\right)$ is the set of nodes in the BN that have an arrow to node $X_{i}$, we get

$$
\begin{aligned}
P\left(A=x \mid O=x, C P_{1}=c_{1}, \ldots, C P_{n}=c_{n}\right)= & \\
=\alpha & \cdot P(A=x) \cdot \\
& \cdot P\left(C P_{1}=c_{1} \mid A=x\right) \cdot \ldots \cdot P\left(C P_{n}=c_{n} \mid A=x\right) . \\
& \cdot P\left(O=x \mid C P_{1}=c_{1}, \ldots, C P_{n}=c_{n}\right)
\end{aligned}
$$

$n$ is the number of available CPs for this type of context, $c_{i}$ the output of the $i$-th CP and $\alpha$ is a scaling factor that normalises the resulting probabilities such that they sum up to 1 . Once $P\left(A \mid O, C P_{1}, \ldots, C P_{n}\right)$ is computed for every possible value of $A$, the one with highest probability is picked and its computed probability can be used as the probability of correctness of the final output.

\subsection{Defining the conditional probabil- ities in the Bayesian network}

Let us now define the condition probabilities in Equation 1 so that we can compute $P\left(A \mid O, C P_{1}, \ldots, C P_{n}\right)$ and thus determine a final output given inputs from multiple CPs.

$P(A)$ can be estimated by observing the frequency of the different final outputs, i.e. we can use as $P(A)$ the past occurrence rate of each value at node $\mathrm{O}$. At the beginning, when there are no or too few outputs of node $\mathrm{O}$ to construct $P(A)$, we can either set $P(A)$ to some standard distribution (e.g. one that takes into account that sitting is a lot more likely than standing), or in general start with all outcomes equiprobable.

$P\left(C P_{i} \mid A\right)$ is the probability of correctness (poc) advertised by the $i$-th $C P$. That is, we assume that each $\mathrm{CP}$ can advertise the probability of each output given the actual context. These values fill what is often called the confusion matrix, for example in $[7,13,4]$. The confusion matrix essentially measures how well a $\mathrm{CP}$ can infer a type of context. Indeed, such a matrix is usually produced when a sensor logic algorithm that 
Table 1. CPT for Example 1.

\begin{tabular}{|c|c|c|c|c|}
\hline$A=s i$ & $C P_{1}$ & $C_{2}$ & $C P_{3}$ & $C P_{4}$ \\
\hline$s i$ & $\mathbf{0 . 8}$ & 0.2 & 0.2 & $\mathbf{0 . 7}$ \\
$s t$ & 0.1 & $\mathbf{0 . 6}$ & 0.3 & 0.2 \\
$l y$ & 0.1 & 0.2 & $\mathbf{0 . 5}$ & 0.1 \\
\hline \hline$A=s t$ & & & & \\
\hline$s i$ & 0.1 & 0.2 & 0.2 & 0.2 \\
$s t$ & $\mathbf{0 . 9}$ & $\mathbf{0 . 6}$ & $\mathbf{0 . 5}$ & $\mathbf{0 . 6}$ \\
$l y$ & 0 & 0.2 & 0.3 & 0.2 \\
\hline \hline$A=l y$ & & & & \\
\hline$s i$ & 0.1 & 0.2 & 0.1 & 0.1 \\
$s t$ & 0.2 & $\mathbf{0 . 6}$ & 0.3 & 0.1 \\
$l y$ & $\mathbf{0 . 7}$ & 0.2 & $\mathbf{0 . 6}$ & $\mathbf{0 . 8}$ \\
\hline
\end{tabular}

produces high-level context from sensor data is tested with real data, as this is a common means of comparing the effectiveness of different algorithms.

A sensible definition of $P\left(O \mid C P_{1}, C P_{2}, \ldots\right)$ is less straightforward and is discussed in the following section. Once we have chosen a definition for $P\left(O \mid C P_{1}, C P_{2}, \ldots\right)$, we can use Equation 1 to combine the $C P$ s to produce a final output.

\subsection{Computing $\mathrm{P}\left(\mathrm{O} \mid \mathrm{CP} \mathrm{P}_{1}, \mathrm{CP}_{2}, \ldots, C \mathrm{P}_{n}\right)$}

Intuitively we want to define $P\left(O \mid C P_{1}, C P_{2}, \ldots, C P_{n}\right)$ such that it takes into account the amount of uncertainty each CP has in its output. In other words, given multiple CPs, the output of CPs that advertise low probability of correctness poc should be considered to a lesser extent than CPs advertising high poc, as the latter are more accurate. We can translate this intuitive strategy into the following formula:

Let $C P$ be the set of all CPs,

$\mathcal{M}$ the set of all possible context values, i.e. all possible values of $A, O$, and $C P_{i}$ are in $\mathcal{M}$,

$c\left(C P_{i} \mid A=a\right)$ the context value output by $C P_{i} \in$

$C P$ when $A=a$ (the true context is $a$ ), i.e.

$P\left(C P_{i}=c\left(C P_{i} \mid A=a\right) \mid A=a\right) \geq P\left(C P_{i}=m \mid A=a\right)$, $\forall m \in \mathcal{M}$.

We define $P\left(O \mid C P_{1}, \ldots, C P_{|C \mathcal{P}|}\right)$ as follows $(|C \mathcal{P}|=n)$ :

$$
\begin{array}{r}
P\left(O=m \mid C P_{1}=c_{1}, \ldots, C P_{|C P|}=c_{|C P|}\right)= \\
=\alpha \prod_{C P_{i} \in \mathcal{C P}} \frac{P\left(C P_{i}=c_{i} \mid A=m\right)}{\sum_{a \in \mathcal{M}} P\left(C P_{i}=c_{i} \mid A=a\right)}
\end{array}
$$

In words, we are taking a product of a value for each CP. For this value, we assume that the true context $A$ is equal to the output $O$ and use the ratio ${ }^{1}$ between the probability of $C P_{i}$ 's output being what it is (given the value of $A$ ) over the sum of probabilities of $C P_{i}$ outputting what it does given all the possible values

${ }^{1}$ If the sum in the ratio turns out to be 0 , we get division by zero. This indicates that this combination of outputs from the CPs cannot occur according to the CPs' conditional probability tables.
Table 2. Extract of node O's output for

\begin{tabular}{|c|c|c|c|c|c|}
\hline$C P_{1}$ & $C P_{2}$ & $\mathrm{CP}_{3}$ & $C P_{4}$ & $O$ & $P\left(O \mid C P_{1}, \ldots, C \bar{P}_{n}\right)$ \\
\hline si & $s i$ & si & si & si & 0.9573 \\
\hline si & si & si & si & st & 0.0342 \\
\hline si & si & si & si & $l y$ & 0.0085 \\
\hline si & st & ly & $s i$ & si & 0.9589 \\
\hline si & st & ly & si & st & 0.0205 \\
\hline$s i$ & $s t$ & ly & $s i$ & ly & 0.0205 \\
\hline st & st & st & st & si & 0.0213 \\
\hline st & st & st & st & st & 0.9574 \\
\hline$s t$ & $s t$ & $s t$ & $s t$ & $l y$ & 0.0213 \\
\hline st & st & $s i$ & ly & $s i$ & 0.037 \\
\hline$s t$ & st & si & ly & $s t$ & 0.6667 \\
\hline st & $s t$ & $s i$ & ly & ly & 0.2963 \\
\hline ly & st & ly & ly & si & 0.0147 \\
\hline ly & st & ly & ly & st & $\approx 0$ \\
\hline ly & $s t$ & by & ly & ly & 0.9853 \\
\hline ly & ly & ly & ly & si & 0.0147 \\
\hline$l y$ & ly & ly & ly & st & $\approx 0$ \\
\hline ly & ly & ly & ly & ly & 0.9853 \\
\hline
\end{tabular}
Example 1 (CPs' CPT is given in Table 1).

of $A . \quad \alpha$ is again the scaling factor that assures that probabilities sum up to 1 as appropriate.

Let us briefly justify why we take the ratio between $P\left(C P_{i}=c_{i} \mid A=m\right)$ and $\sum_{a \in M} P\left(C P_{i}=c_{i} \mid A=a\right)$ in Definition 2. In words, this means that we take each CP's output in the context of how likely it is output for each and every possible value of $A$. So, if for example $C P_{i}$ nearly always outputs $c_{i}$ only when the actual context $A$ is $m$ then-without knowledge of $A-C P_{i}$ 's output being $c_{i}$ contributes much to our belief that the actual context is $m$. However, if on the other hand $C P_{i}$ is equally likely to output $c_{i}$ for every possible actual context $A$, then $C P_{i}$ 's output being $c_{i}$ will be useless. The following example should better illustrate the behaviour of Definition 2.

Example 1. Consider an example scenario of activity detection which is meant to be to some extent realistic, but is also constructed to better show the desirable behaviour of Def. 2. In this example, the possible values of the detected activity context $A$ are sitting (si), standing $(s t)$ and lying $(l y)$. Table 1 shows the conditional probability table (CPT) for the four CPs in this example. The table tell us, given each actual activity, with what probability each possible value is output by a CP. The numbers in bold represent the probability of the value that a $\mathrm{CP}$ is most likely to output given an actual context. For example, given that the actual con- 
text is $A=s i, C P_{1}$ 's most likely output is correctly the context value $s i$ with probability 0.8 . Instead, given $A=s i, C P_{2}$ 's most likely output is erroneously st with probability 0.6 .

Table 2 shows an extract of node O's probability table when we apply Def. 2. Let us now discuss these outputs. For example, given the input $(s i, s t, l y, s i)$ from the four CPs, the most likely output is $O=s i$. This reflects the fact that, in Table 1 , given an activity $A=s i$, the most likely value of each CP is $(s i, s t, l y, s i)$ as emphasised by the probabilities in bold. This behaviour is also obtained in the cases $(s t, s t, s t, s t)$ and $(l y, s t, l y, l y)$ for the activities $A=s t$ and $A=l y$, respectively, as expected. Further, the probability of $O$ 's output is greater than the poc of any single CP, so taking all four CP's into account does give us more certainty about the cor= rectness of the output than using any single $\mathrm{CP}$.

It may at first seem surprising that the final outputs are correct with such high probability $(>0.9$ for most cases) considering that of the four CPs, only two actually output the correct value with highest probability $\left(C P_{1}\right.$ and $\left.C P_{4}\right)$ while $C P_{2}$ is completely useless, as it always has the same output probability distribution regardless of the actual context (it is as if it had no sensors at all to detect the actual context and just outputs the three context values according the probabilities in Table 1). The reason is that, since $C P_{2}$ is not useful, it does not contribute-either positively or negatively - to O's output. The ratio in Def. 2 between $P\left(C P_{i}=c_{i} \mid A=m\right)$ and $\sum_{a \in \mathcal{M}} P\left(C P_{i}=c_{i} \mid A=s i\right)$ achieves that effect, as for every value $s i, s t, l y$, that $A$ can take, the ratio for $C P_{2}$ is always the same (equal to $\frac{1}{3}$ ). In other words, as $\mathrm{CP}_{2}$ 's output is in no way conditional on the actual context, each output is therefore equally likely regardless of what the actual context is (e.g. $C P_{2}$ outputs st with probability 0.6 regardless of what $A$ is). Another effect of the ratio in Def. 2 is that $C P_{3}-$ while not outputting the correct value with highest probability in the cases of $A=s i$ and $A=l y$-is nevertheless useful. This is because it has a strong bias towards some value (what this value is is not important), and this bias is conditional on the actual context. Thus, the output of $C P_{3}$, while incorrect, gives us information about the actual context. To understand this, consider a similar case where a person must answer yes/no questions and we know that the person will always lie. In that case, each answer, while incorrect, allows us to infer the correct answer. The trick lies in knowing that the person lies, which in our case translates into the assumption that the CPs are reliable in their advertised poc, regardless of whether the output is correct.

We have also included in the table a case where the three CPs that have an influence on the output $\left(C P_{1}\right.$, $\mathrm{CP}_{3}$ and $\mathrm{CP}_{4}$ ) produce conflicting values. In the case shown in the table, the CPs' output is (st, st, si, ly), with a final output of st. This makes sense because $C P_{1}$ is very accurate and there is a very low probability that it will output st when the actual context is anything
Table 3. A possible distribution for $P(A)$ and resulting $P(A \mid O)$ for Example 1.

\begin{tabular}{|l|c|}
\hline$A$ & $P(A)$ \\
\hline si & 0.6 \\
st & 0.2 \\
$l y$ & 0.2 \\
\hline
\end{tabular}

\begin{tabular}{|c|c|c|}
\hline$O$ & $A$ & $P(A \mid O)$ \\
\hline$s i$ & $s i$ & $\mathbf{0 . 9 6 9 4}$ \\
si & st & 0.0153 \\
$s i$ & $l y$ & 0.0153 \\
\hline$s t$ & $s i$ & 0.1194 \\
$s t$ & $s t$ & $\mathbf{0 . 8 4 0 8}$ \\
st & $l y$ & 0.0398 \\
\hline ly & si & 0.1194 \\
ly & st & 0.0398 \\
ly & ly & $\mathbf{0 . 8 4 0 8}$ \\
\hline
\end{tabular}

other than st. Therefore, its opinion weighs more than that of $\mathrm{CP}_{4}$ which is less accurate and outputs the conflicting context $l y$. However, this conflict contributes to the low bias of the output's probabilities, indicating that there is greater uncertainty in the output. We will come back to this case in Section 3.3.

In the above example, we have discussed the behaviour of Def. 2 for $P\left(O \mid C P_{1}, C P_{2}, \ldots, C P_{n}\right)$. This could be used directly to determine the final output if the prior $P(A)$ is not known. On the other hand, if a good estimate of $P(A)$ is available, we can instead use $P\left(O \mid C P_{1}, C P_{2}, \ldots, C P_{n}\right)$ to compute $P\left(A \mid O, C P_{1}, \ldots, C P_{n}\right)$, which additionally takes into account the likelihood of each context value. Table 3 (right) shows the resulting probabilities for $P(A \mid O)^{2}$, given the prior of $A$, i.e. $P(A)$, in Table 3 (left). Using the latter often does not change the most likely output (i.e. the value with highest probability), but it does change the resulting probability of correctness, as it better reflects the true probability of correctness given the likelihood of each possible value of $A$.

\section{Further dependencies}

The Bayesian network (BN) we described in Figure 1 appears a reasonable generic model for combining data from multiple CPs. However, in practice, there are often details which are not modelled by this BN. For instance, in the activity example, the prior distribution of the actual activity $P(A)$ may be directly influenced by the location. To see this, consider the following example of a smart home. Suppose the three possible activities detected by the CPs are $\mathcal{M}=$ \{sitting, standing, lying $\}$. Further, assume we have a smart home that consists of four rooms: a bedroom, a living room, a kitchen and a bathroom. If there is no workplace (e.g. desk) in the bedroom, it is fair to

\footnotetext{
${ }^{2}$ We report $P(A \mid O)$ instead of $P\left(A \mid O, C P_{1}, \ldots, C P_{n}\right)$ simply for compactness. Also, as $P(A \mid O)$ is simply a weighted average of $P\left(A \mid O, C P_{1}, \ldots, C P_{n}\right)$ over all possible combinations of $C P$ values, it is in a sense a summary of $P\left(A \mid O, C P_{1}, \ldots, C P_{n}\right)$.
} 


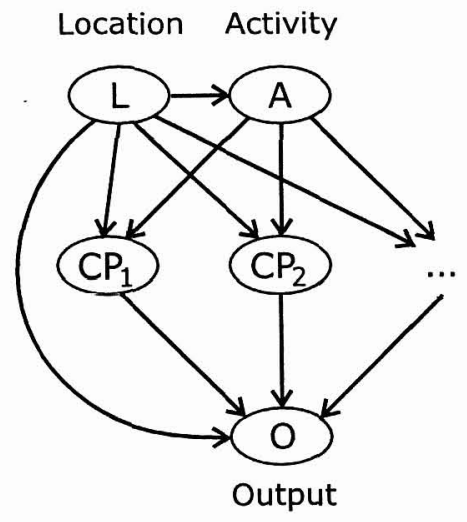

(a) Case I: CPs use L.
Location Activity

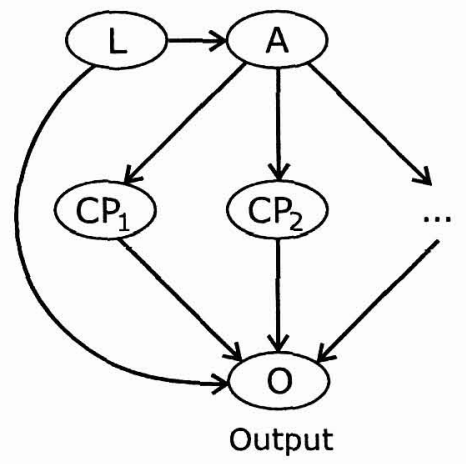

(b) Case II: CPs do not use L.

Figure 2. Two versions of a Bayesian network that also takes into account available location information. Figure 2(a) (described in Section 3.1) considers the case where the CPS for context type $A$ are capable of using the additional context L. Figure 2(b) (described in Section 3.2) considers the case where the CPs cannot use the additional context $L$.

believe that the most likely activity in the bedroom is lying. Similarly, if there are no chairs in the kitchen (e.g. because there is little space) and as a result dinner is served in the living room, the most likely activity in the kitchen will be standing. In the living room, where we may be dining, watching TV, or reading a book (which we might do lying on the sofa), the most likely activity would be sitting, followed by lying.

Table 4 shows a possible set of probability distributions of the activity given the location. These may be statistical results of observing the home inhabitant during a training phase of the system to detect his location patterns. In our case, these values are made up, but try to realistically capture the typical behaviour of a person at home. Notice that there are no probabilities equal to zero. This is done on purpose: even if during the training phase we have never observed the user lying in the kitchen, we must set the probability for every case that could eventually occur to a non-zero value. Otherwise, the Bayesian network will never allow that value to be output. So, if the user should ever be lying on the floor in the kitchen, unable to call for help, it is important for the smart home to be able to detect his condition, however unlikely, and automatically call for help. Indeed, it is common in Bayesian networks to add a small uniform baseline distribution to CPTs to avoid zero-probability values, and thus allow for values that did not occur in the training phase.

The new dependency of the activity on location can be modelled in the BN by introducing a new node $\mathrm{L}$ representing the current location of the user. Consequently, because this node does not depend on any parent nodes, we need to define its prior $P(L)$. In our smart home example, this would be the probability of the user being in a specific location at any time. An example distribution is given in Table 5. Assume that the person is out of the house during the day, only spending time at home from evening to morning. In the specific case of Table 5, half of the time at home is spent in the bedroom (mostly sleeping at night), a considerable time is spent in the living room (dinner, breakfast, watching TV and reading), with a small amount of time in the kitchen (cooking and washing up), and an even smaller amount of time in the bathroom.

In the following two sections, we look at howgiven that the activity is location-dependent-the final output can depend on the location (additionally to the output of the activity CPs). We distinguish between two cases: the case where the activity CPs are aware and are able to take advantage of the current location information, and the opposite case where they have no knowledge and thus do not use the current location.

\subsection{Case I: Activity CPs use location information}

Assume that some or all activity CPs can use the location information to affect their output. For instance, given the location "bedroom" and the high likelihood of lying, a CP might switch to an algorithm that is less good at detecting lying (which occurs often anyway) but can better distinguish standing and sitting from lying. The $\mathrm{BN}^{3}$ is illustrated in Figure 2(a). Now, there is a new node $L$ representing the random variable for location. The activity is now location-dependent, and so there is an arrow $L \rightarrow A$. There is also an arrow from $L$ to each $C P$, indicating that now CPs use location to improve their accuracy. We have also added an arrow

${ }^{3}$ If a $\mathrm{CP}$ does not use location information, it is equivalent to a $\mathrm{CP}$ that is conditional on the location, but has the same probability distribution for its output for every value of location. Thus, we can build a Bayesian network where every $\mathrm{CP}$ depends on the location, even if some CPs do not actually use this information. 
Table 4. Example CPT for locationdependent activity in a smart-home.

\begin{tabular}{|c|c|c|c|c|}
\cline { 2 - 5 } \multicolumn{1}{c|}{$P(A \mid L)$} & \multicolumn{4}{c|}{ Location } \\
\hline Activity & bedroom & living r. & kitchen & bathroom \\
\hline sitting & 0.1 & 0.6 & 0.01 & 0.25 \\
standing & 0.1 & 0.1 & 0.98 & 0.65 \\
lying & 0.8 & 0.3 & 0.01 & 0.01 \\
\hline
\end{tabular}

\section{Table 5. Example probability distribution} for location in a smart-home.

\begin{tabular}{c|cccc}
$L$ & bedroom & living room & kitchen & bathroom \\
\hline$P(L)$ & 0.5 & 0.39 & 0.07 & 0.04
\end{tabular}

$\mathrm{L} \rightarrow \mathrm{O}$, which we will justify shortly. Table 4 gives a possible CPT for A. L itself must be given a prior distribution, and an example of this is given in Table 5 .

Given this BN, it turns out that the output strategy does not need to change. We can still apply Def. 2 to determine $\mathrm{P}\left(\mathrm{O} \mid \mathrm{CP} \mathrm{P}_{1}, \mathrm{CP}_{2}, \ldots, C \mathrm{CP}_{n}\right)$. Only its notation must change slightly. Let us look at the details.

First of all, because the CPs' output is now influenced by knowledge of the user's location, their advertised probability of correctness is now $P\left(C P_{i} \mid A, L\right)$. This is also the CPT table for each CP in the BN. Now, we must define the new strategy for the final output, i.e. the CPT for node O. This output is based on the output of the CPs, as before, plus the additional user location, and can be defined as follows:

$$
\begin{array}{r}
P\left(O=m \mid C P_{1}=c_{1}, \ldots, C P_{|C P|}=c_{\mid C P}, L=l\right)= \\
=\alpha \prod_{C P_{i} \in C \mathcal{P}} \frac{P\left(C P_{i}=c_{i} \mid A=m, L=l\right)}{\sum_{a \in \mathcal{M}} P\left(C P_{i}=c_{i} \mid A=a, L=l\right)}
\end{array}
$$

$P\left(C P_{i}=c_{i} \mid A=m, L=l\right)$ is the poc advertised by $C P_{i}$, which is conditional on both activity and location. Comparing this definition with Def. 2, notice that the operation to calculate the output is still the same: we have a division for each $C P_{i}$ of its probability of its output conditional on the activity that $O$ would output $P\left(C P_{i}=c_{i} \mid A=m, L=l\right)$ by the sum of this probability for every possible value of $A$, $\sum_{a \in \mathcal{M}} P\left(C P_{i}=c_{i} \mid A=a, L=l\right)$. The fact that these probabilities are conditional on the location does not change the calculation: $O$ knows the location information, but does not directly use it. It is the CPs that use this location information in producing their output and poc. Therefore, although the final output $\mathrm{O}$ does not take into account location directly, its output does indirectly take it into account, as the CPs take location information into account in their own output.

If $O$ does not appear to directly depend on $L$, why then did we insert the arrow $L \rightarrow O$ in the BN? The reason is that, to compute $\mathrm{O}$ using the formula above, we need to have the complete CPT of each $\mathrm{CP}$, i.e. $P\left(C P_{i}=c_{i} \mid A=m, L=l\right)$, which is the CP's advertised poc for each case that the $\mathrm{CP}$ distinguishes. Further, $\mathrm{O}$ needs to know the current location in order to determine which values to pick from $C P_{i}$ 's $\mathrm{CPT}$, as these values are location-dependent. So, from a Bayesian point of view, $\mathrm{O}$ does indeed depend directly on $\mathrm{L}$, because its output changes depending on the value of $L$.

Finally, we can compute the probability of correctness (poc) of the final output $P\left(A \mid O, C P_{1}, \ldots, C P_{n}, L\right)$, whose formula can be obtained directly from the Bayesian net by applying Bayes' rule (cf. Def. 1 for the simple case without the node $\mathrm{L}$ ):

$$
\begin{aligned}
P(A= & \left.x \mid O=x, C P_{1}=c_{1}, \ldots, C P_{n}=c_{n}, L=l\right)= \\
=\alpha & \cdot P(L=l) \cdot P(A=x \mid L=l) . \\
& \cdot P\left(C P_{1}=c_{1} \mid A=x, L=l\right) \cdot \ldots \cdot P\left(C P_{n}=c_{n} \mid A=x, L=l\right) . \\
& \cdot P\left(O=x \mid C P_{1}=c_{1}, \ldots, C P_{n}=c_{n}, L=l\right)
\end{aligned}
$$

\subsection{Case II: Activity CPs do not use location information}

Consider now the case where the CPs are not designed to take location information into account when it is available. In this case, if there is location information available about the user through some positioning system, the component producing the final output can take this location data into account to improve the accuracy of its output. We extend our BN accordingly in Figure 2(b). The CPs do not depend on location, i.e. their output probability distribution is independent of the location, and conditional only on the current activity, while the final output does bias its output based on location information. Thus, we have only the new edges $L \rightarrow A$ and $L \rightarrow O$ in the BN. As a strategy for computing the output of $\mathrm{O}$, we can use the following:

$$
\begin{aligned}
& P\left(O=m \mid C P_{1}=c_{1}, \ldots, C P_{|C P|}=c_{|C \mathcal{P}|}, L=l\right)= \\
& =\alpha \prod_{C P_{i} \in C \mathcal{P}}\left(\frac{P\left(C P_{i}=c_{i} \mid A=m\right)}{\sum_{a \in \mathcal{M}} P\left(C P_{i}=c_{i} \mid A=a\right)}\right) \cdot \frac{P(A=m \mid L=l)}{\sum_{k \in \mathcal{L}} P(A=m \mid L=k)}
\end{aligned}
$$

where $\mathcal{L}$ is the set of possible locations.

This definition works in the following way, it takes Def. 2 -i.e. the old formula for computing the final output when the CPs have no knowledge of (or do not use) location - and weighs this by the probability of this output occurring given the current location, which is the ratio $\frac{P(A=m \mid L=l)}{\sum_{k \in L} P(A=m \mid L=k)}$ in Def. 5. This term is calculated by dividing the probability of this output occurring at this location over the total probability of this output occurring everywhere. Def. 5 is perhaps best understood with an example.

For completeness, we first provide the probability of correctness of the final output for this case, which 
again results from applying Bayes' product rule:

$$
\begin{aligned}
P\left(A=x \mid O=x, C P_{1}=c_{1}, \ldots, C P_{n}=c_{n}, L=l\right)= & \text { (6) } \\
=\alpha & \cdot P(L=l) \cdot P(A=x \mid L=l) . \\
& \cdot P\left(C P_{1}=c_{1} \mid A=x\right) \cdot \ldots \cdot P\left(C P_{n}=c_{n} \mid A=x\right) . \\
& \cdot P\left(O=x \mid C P_{1}=c_{1}, \ldots, C P_{n}=c_{n}, L=l\right)
\end{aligned}
$$

Example 2. Let's continue with the smart home scenario of Example 1, i.e. consider a four-room home with the location distribution of Table 5 and the location-dependent activity distribution of Table 4 . Further, assume there are four CPs for activity and let us take the probability of correctness for their output from Example 1. Recall that in this CPs' $\mathrm{CPT}, \mathrm{CP}_{2}$ is useless and does not affect the final output.

We now compute the output $O$ with Def. 5. Tables 6-7 show an extract of the results, which we now discuss by comparing the output of the four CPs with the output of node O. Keep in mind that what $C_{2}$ outputs is irrelevant, as it provides no useful information, and therefore the final output is the same when we change $\mathrm{CP}_{2}$ 's output keeping the others fixed.

Consider the first table (in Table 6) where the location is the bedroom. According to Table 4, lying is very likely with sitting and standing unlikely. Looking at the results, we see for example that when the four CPs output the values $(l y, s i$, st, si), i.e. only one of the four CPs output the correct context value, the final output is very clearly lying (with probability 0.8365 ). This results from the fact that at this location lying is much more likely than the other two activities. The same thing happens when the output of the CPs is (st, $s t, s i, l y$ ), with a final output of lying with probability 0.7758 . When all four CPs output lying, the probability of the correct output is nearly $1(0.9971$ in the table), which again makes sense considering the high likelihood of lying in the bedroom.

Let us now move on to the living room. Here the most likely activity is sitting (see Table 4), with lying half as likely and standing very unlikely. So, when only $\mathrm{CP}_{4}$ outputs sitting (with the other CPs outputting either standing or lying), the final output is sitting, recognising the high likelihood of this activity in the living room. Yet, should all CPs output lying, the final output is lying with a probability of nearly $1(0.9555$ in the table). This is partly caused by the fact that the probability of lying in the living room is substantial $(0.3)$, even if only half of sitting (0.6). It is also partly caused by the probability of lying in the living room being quite high compared to other rooms in the home, particularly the kitchen and bathroom where it is very unlikely.

Moving on to the kitchen, the most likely activity is now standing (Table 4), with the other two activities being very unlikely (we are assuming that the home inhabitant eats in the living room). Notice here that if all ${ }^{4}$ four CPs output lying or sitting, which are very un-

\footnotetext{
${ }^{4} \mathrm{Recall}$ that what $\mathrm{CP}_{2}$ outputs is irrelevant.
}

likely activities in the kitchen, the final output agrees with the CPs. However, in the case of the four CPs' outputs being sitting, the probability of sitting is not particularly high $(0.6768)$. This is because standing is a much more likely activity in the kitchen, and therefore even when all CPs output sitting there is still a reasonable probability that the activity is actually standing. However, in the case of all CPs outputting lying, the final output lying is extremely likely (0.9773) while standing's probability is completely zero. To understand this, we must observe that in the CPs' CPT (Table 1), $C P_{1}$ 's probability of outputting lying when the actual activity is standing is exactly zero. Thus, whenever $C P_{1}$ outputs lying, and assuming that the CPs are completely reliable in their advertised poc values, we must conclude that it is not possible for the actual activity to be standing. This in turn allows the probability of lying to be very high.

Finally, looking at the outputs for the bathroom (Table 7), we see that if all CPs output lying, then the final output will also be lying with about twice the probability $(0.6323)$ of sitting $(0.3677)$, despite the fact that lying is very unlikely in the bathroom. This is an important behaviour for a context provision component in the smart home scenario, because an elderly person may slip in the bathroom and fall in the bathtub or onto the floor (in a lying position) and be unable to call for help to get up. Thus, while on one hand the addition of location information improves the bias of the final output probabilities when there is weak bias in the $\mathrm{CPs}$ (which is similar to saying that the CPs have small poc), on other hand the presence of multiple CPs for the same context helps us correctly determine with an improved amount of certainty also those context values that are very unlikely, but whose correct detection is crucial to the smart home application.

For lack of space, we omit a discussion on $P(A \mid O, L)$ (Def. 6), which essentially weighs the output of $O$ on the likelihood of each context $A$ and location $L$.

\subsection{Taking poc of $L$ into account}

Up to now, we have assumed that the location information is accurate. However, in practice this information will usually be provided by a positioning system with a limited probability of correctness (poc) of its own. Thus, we now want to also take into account the advertised poc of node L. The Bayesian networks for cases I and II need to updated. Figure 3 shows the new Bayesian networks. Here, the activity depends on the actual location $A L$, which is measured and output by $L$ with a probability of correctness $\operatorname{poc}(L)$. O uses the measured location to output the final activity. For case I, the CPs' CPT also depends on L, as the CPs are capable of using available location information to improve their output.

Given that $L$ comes with its own poc, how should we modify the strategy for computing $P\left(O \mid C P_{1}, \ldots, C P_{n}, L\right)$ ? 
Table 7. Continuation of Table 6.

Table 6. Final output probabilities for Example 2 ( $s i=$ sitting, $s t=s t a n d i n g, l y=l y i n g$ ). The values in bold are the values actually output.

\begin{tabular}{|c|c|c|c|c|c|}
\hline \multicolumn{6}{|c|}{ bedroom } \\
\hline$C P_{1}$ & $C P_{2}$ & $C P_{3}$ & $C P_{4}$ & $O$ & $P\left(O \mid C P_{1}, \ldots, C P_{4}, L\right)$ \\
\hline si & si & si & $s i$ & si & 0.9371 \\
\hline si & si & $s i$ & si & st & 0.0201 \\
\hline si & $s i$ & si & si & ly & 0.0428 \\
\hline ly & si & st & si & si & 0.1635 \\
\hline ly & si & st & $s i$ & st & 0 \\
\hline ly & $s i$ & st & $s i$ & ly & 0.8365 \\
\hline st & st & $s i$ & ly & $s i$ & 0.019 \\
\hline st & st & si & ly & $s t$ & 0.2052 \\
\hline st & st & si & ly & ly & 0.7759 \\
\hline ly & ly & ly & ly & $s i$ & 0.0029 \\
\hline ly & ly & ly & ly & st & 0 \\
\hline ly & ly & ly & ly & ly & 0.9971 \\
\hline \multicolumn{6}{|c|}{ living room } \\
\hline$C P_{1}$ & $\mathrm{CP}_{2}$ & $C P_{3}$ & $\mathrm{CP}_{4}$ & 0 & $P\left(O \mid C P_{1}, \ldots, C P_{4}, L\right)$ \\
\hline$s i$ & si & $s i$ & si & si & 0.9936 \\
\hline si & $s i$ & $s i$ & si & st & 0.0036 \\
\hline si & si & si & si & ly & 0.0028 \\
\hline ly & st & st & si & si & 0.7577 \\
\hline ly & st & st & si & st & 0 \\
\hline ly & st & $s t$ & si & ly & 0.2423 \\
\hline st & ly & ly & si & si & 0.7909 \\
\hline st & ly & ly & si & st & 0.1224 \\
\hline st & ly & ly & si & ly & 0.0867 \\
\hline ly & ly & ly & ly & si & 0.0445 \\
\hline ly & ly & ly & ly & st & 0 \\
\hline ly & ly & ly & ly & ly & 0.9555 \\
\hline \multicolumn{6}{|c|}{ kitchen } \\
\hline$C P_{1}$ & $\mathrm{CP}_{2}$ & $C P_{3}$ & $\mathrm{CP}_{4}$ & $O$ & $P\left(O \mid C P_{1}, \ldots, C P_{4}, L\right)$ \\
\hline$s i$ & $s i$ & $s i$ & si & si & 0.3214 \\
\hline si & si & si & si & st & 0.6768 \\
\hline si & si & si & si & ly & 0.0018 \\
\hline st & st & st & st & si & 0.0004 \\
\hline st & st & st & st & st & 0.9994 \\
\hline st & st & st & st & ly & 0.0002 \\
\hline ly & ly & ly & ly & si & 0.0227 \\
\hline ly & ly & ly & ly & st & 0 \\
\hline ly & ly & ly & $l y$ & ly & 0.9773 \\
\hline
\end{tabular}

\begin{tabular}{|c|c|c|c|c|c|}
\hline \multicolumn{6}{|c|}{ bathroom } \\
\hline$C P_{1}$ & $C P_{2}$ & $C P_{3}$ & $\mathrm{CP}_{4}$ & $O$ & $P\left(O \mid C P_{1}, \ldots, C P_{4}, L\right)$ \\
\hline$s i$ & si & si & $s i$ & si & 0.9469 \\
\hline$s i$ & $s i$ & $s i$ & $s i$ & st & 0.0529 \\
\hline si & si & si & si & ly & 0.0002 \\
\hline st & $s i$ & si & si & si & 0.1989 \\
\hline st & $s i$ & $s i$ & si & st & 0.8004 \\
\hline st & si & $s i$ & si & ly & 0.0007 \\
\hline ly & ly & st & $s i$ & si & 0.9751 \\
\hline ly & ly & st & si & st & 0 \\
\hline ly & ly & st & si & ly & 0.0249 \\
\hline ly & ly & ly & ly & si & 0.3677 \\
\hline ly & ly & ly & ly & st & 0 \\
\hline ly & ly & ly & ly & ly & 0.6323 \\
\hline
\end{tabular}

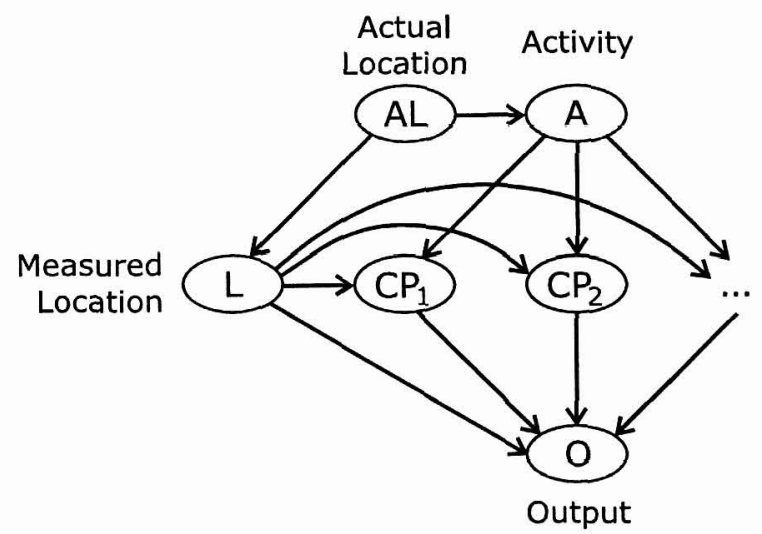

(a) Updated BN for case I.

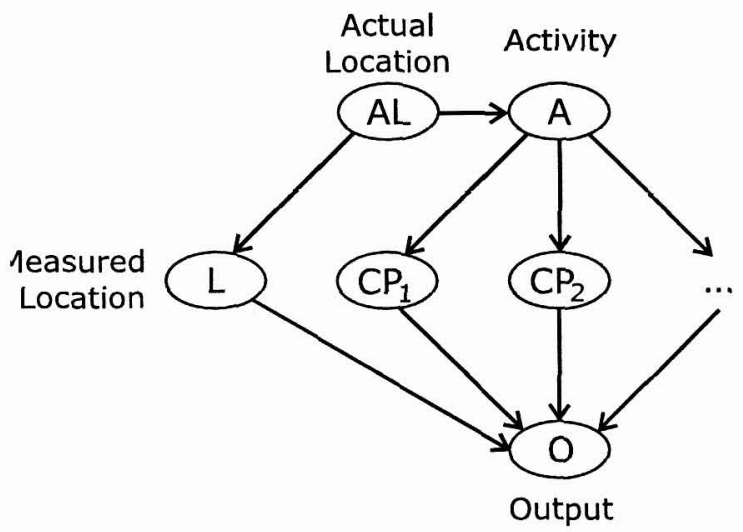

(b) Updated BN for case II.

Figure 3. Updated version of BN in Figure 2. Now, the actual location AL is measured and output by $L$ with an advertised probability of correctness $\operatorname{poc}(L)$. 
$P(O \mid C P s, L)$ for different poc $(L)$ values when CPs' output is (st, st, si, ly) and location is "bedroom"

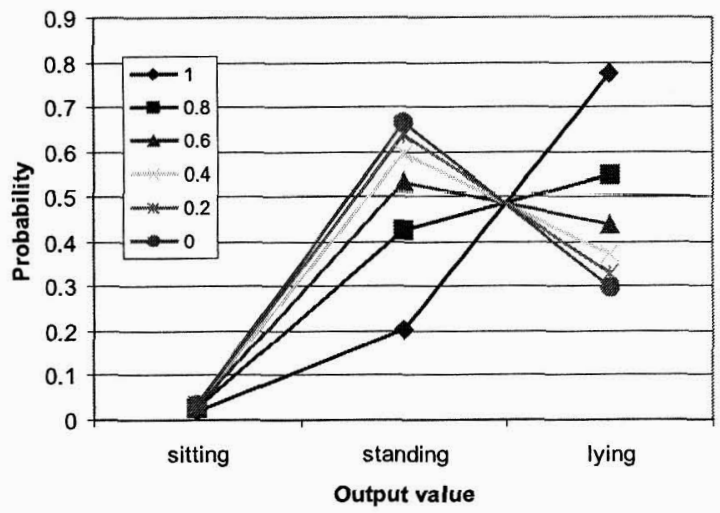

Figure 4. Final output probabilities $P\left(O \mid C P_{1}, \ldots, C P_{n}, L\right)$ with varying $\operatorname{poc}(L)$ when $L=$ bedroom and CP's output is (standing, standing, sitting, lying).

\subsubsection{Case II}

Let us look at case II first, as it results in a simpler expression. We can introduce L's poc in Def. 2 by introducing a linear dependency on L's poc between two extremes. These two extremes are:

1. When L's $p o c$ is equal to 1 , we want $P\left(O \mid C P_{1}, \ldots, C P_{n}, L\right)$ to use Def. 5 , where we assumed that $\mathrm{L}$ was completely accurate.

2. When L's poc is equal to 0 , we want $P\left(O \mid C P_{1}, \ldots, C P_{n}, L\right)$ to use Def. 2$)$, where we had no location information at all, i.e. when the location information is completely inaccurate, we will ignore it completely.

We now apply a linear dependency $f(x)=a x+b$ to these two extreme cases. We omit the details for lack of space, and just provide the resulting expression:

$$
\begin{aligned}
P\left(O=m \mid C P_{1}=c_{1}, \ldots, C P_{|C \mathcal{P}|}=c_{|C \mathcal{P}|}, L=l\right)= & (7) \\
=\alpha & \prod_{C P_{i} \in C \mathcal{P}} \frac{P\left(C P_{i}=c_{i} \mid A=m\right)}{\sum_{a \in \mathcal{M}} P\left(C P_{i}=c_{i} \mid A=a\right)} \cdot \\
& \cdot\left[\left(\frac{P(A=m \mid L=l)}{\sum_{k \in \mathcal{L}} P(A=m \mid L=k)}-1\right) \cdot P(L=l \mid A L=l)+1\right]
\end{aligned}
$$

Let us continue with Example 2. Specifically, let us look at the specific case where we are in the bedroom and the four CPs output (st, st, si, ly) (in Table 6). Figure 4 shows the probability of the three output values when we vary the poc of the location information. When the location information is believed to be accurate and the location provider advertises $\operatorname{poc}(L)=1$ for every location, then we have the results we previously showed in Table 6 . In Figure 4, it is the line labelled 1 . In this case, the most likely output is lying (because we are in the bedroom). When $\operatorname{poc}(L)=0$, then we have the results without considering at all location information, which we compiled in Table 2 . In Figure 4 , it is the line labelled 0 . In this case, the most likely output is sitting. In the chart, we also see the output probabilities for different values of $\operatorname{poc}(L)$, where for simplicity we assume that every location has the same $\operatorname{poc}(L)$. There is a threshold point at around $\operatorname{poc}(L)=0.7$, where below 0.7 the most likely output is sitting, whereas above 0.7 it is lying. Therefore, if we have no location information, then given the conflicting CP output ( $s t, s t, s i, l y$ ) we will assume we are sitting. But if we know we are in the bedroom, then it is more likely that we are lying. This is a good example of how the introduction of an additional dependency improves the quality of the final output when there is inaccurate data from the CPs.

\subsubsection{Case I}

Let us now consider case I (i.e. where the activity CPs use location information). We can apply the same technique of a linear dependence between two extremes, which are:

1. When L's $p o c$ is equal to 1 , we want $P\left(O \mid C P_{1}, \ldots, C P_{n}, L\right)$ to use Def. 3 , where we assumed that $L$ was completely accurate.

2. When L's $p o c$ is equal to 0 , we want $P\left(O \mid C P_{1}, \ldots, C P_{n}, L\right)$ to use Definition 2, where we had no location information at all, i.e. when the location information is completely inaccurate, we will ignore it completely.

Let $p$ be the expression of Definition 3 and $q$ the expression of Definition 2, i.e.

$$
\begin{gathered}
p:=\prod_{C P_{i} \in C \mathcal{P}} \frac{P\left(C P_{i}=c_{i} \mid A=m, L=l\right)}{\sum_{a \in \mathcal{M}} P\left(C P_{i}=c_{i} \mid A=a, L=l\right)} \\
q:=\prod_{C P_{i} \in C \mathcal{P}} \frac{P\left(C P_{i}=c_{i} \mid A=m\right)}{\sum_{a \in \mathcal{M}} P\left(C P_{i}=c_{i} \mid A=a\right)}
\end{gathered}
$$

Applying the linear dependency, we get:

$$
\begin{aligned}
P(O & \left.=m \mid C P_{1}=c_{1}, \ldots, C P_{|C P|}=c_{|C P|}, L=l\right)= \\
& =(p-q) \operatorname{poc}(l)+q=p \cdot \operatorname{poc}(l)-q(1-\operatorname{poc}(l))
\end{aligned}
$$

$q$ uses $P\left(C P_{i} \mid A\right)$, but the CPs advertise only their output conditional also on the location $P\left(C P_{i} \mid A, L\right)$. However, $P\left(C P_{i} \mid A\right)$ can be computed by applying Bayes' rule on the dependencies in the Bayesian net:

$$
\begin{aligned}
& P\left(C P_{i}=c_{i} \mid A=m\right)= \\
& =\sum_{l_{1} \in \mathcal{L}} P\left(C P_{i}=c_{i} \mid A=m, L=l_{1}\right) \underbrace{P\left(L=l_{1}\right)}_{\sum_{l_{2} \in \mathcal{L}} P\left(L=l_{1} \mid A L=l_{2}\right) P\left(A L=l_{2}\right)} \\
& =\sum_{l_{1} \in \mathcal{L}} P\left(C P_{i}=c_{i} \mid A=m, L=l_{1}\right) \sum_{l_{2} \in \mathcal{L}}^{P}\left(L=l_{1} \mid A L=l_{2}\right) P\left(A L=l_{2}\right)
\end{aligned}
$$


where $P\left(C P_{i} \mid A, L\right), P(L \mid A L)$ and $P(A L)$ are given in the Bayesian net. Furthermore, $\operatorname{poc}(l)$ can be read from $L$ 's CPT, using $\operatorname{poc}(l):=P(L=l \mid A L=l)$. Thus, the whole resulting formula is:

Let $\sigma:=\sum_{l_{2} \in \mathcal{L}} P\left(L=l_{1} \mid A L=l_{2}\right) P\left(A L=l_{2}\right)$ in $P\left(O=m \mid C P_{1}=c_{1}, \ldots, C P_{|C P|}=c_{|C P|}, L=l\right)=$ $=\alpha \prod_{C P_{i} \in \mathcal{C} \mathcal{P}} \frac{P\left(C P_{i}=c_{i} \mid A=m, L=l\right)}{\sum_{a \in M} P\left(C P_{i}=c_{i} \mid A=a, L=l\right)} P(L=l \mid A L=l)+$

$-\prod_{C P_{i} \in C \mathscr{P}} \frac{\sum_{a \in \mathcal{M}} \sum_{l_{1} \in \mathcal{L}} P\left(C P_{i}=c_{i} \mid A=m, L=l_{1}\right) \cdot \sigma}{l_{l^{\prime}} P\left(P_{i}=c_{i} \mid A=a, L=l_{1}\right) \cdot \sigma}(1-P(L=l \mid A L=l))$

\subsubsection{Updated $P(A \mid O, L)$}

Now that the BN has changed, we must also update the resulting $P(A \mid O, L)$, based on the poc of $\mathrm{L}$. This is again done by applying Bayes' rule to the Bayesian network to obtain the new definition of $P(A \mid O, L)$ for the two cases:

Case I:

$$
\begin{aligned}
& P\left(A=x \mid O=x, C P_{1}=c_{1}, \ldots, C P_{n}=c_{n}, L=l\right)= \\
& =\alpha \sum_{k \in \mathcal{L}} P(A L=k) \cdot P(L=l \mid A L=k) \cdot P(A=x \mid L=l) . \\
& \cdot P\left(C P_{1}=c_{1} \mid A=x, L=l\right) \cdot \ldots \cdot P\left(C P_{n}=c_{n} \mid A=x, L=l\right) . \\
& \cdot P\left(O=x \mid C P_{1}=c_{1}, \ldots, C P_{n}=c_{n}, L=l\right)
\end{aligned}
$$

Case II:

$$
\begin{gathered}
P\left(A=x \mid O=x, C P_{1}=c_{1}, \ldots, C P_{n}=c_{n}, L=l\right)= \\
=\alpha \sum_{k \in \mathcal{L}} P(A L=k) \cdot P(L=l \mid A L=k) \cdot P(A=x \mid L=l) . \\
\cdot P\left(C P_{1}=c_{1} \mid A=x\right) \cdot \ldots \cdot P\left(C P_{n}=c_{n} \mid A=x\right) . \\
\cdot P\left(O=x \mid C P_{1}=c_{1}, \ldots, C P_{n}=c_{n}, L=l\right)
\end{gathered}
$$

\section{Conclusions}

We have described a probabilistic mechanism for combining the output of multiple sources of the same context type into a single output that takes into account the advertised probability of correctness of each context provider. Our approach only works with discrete context types, but this includes those context types that can easily be discretised into a small set of values.

While we applied the approach to an abstract smart home example, we have yet to experiment with reallife physical sensors and algorithms to see how well it works in practice. A critical issue are the advertised probability of correctness values of the providers. The mechanism we have described only works well under the assumption that these quality values can be accurately estimated by the context providers. Further, we assume no malicious entities, which could break this technique by advertising high probability of correctness and delivering wrong context data on purpose. These are still areas of future work.
Finally, the smart-home example we described shows that a type of context such as activity can be highly biased by the knowledge of another context, in particular the current location in the home, and we have shown how knowledge of a context type such as location can be incorporated to improve the accuracy (9) in the output of another context type such as activity.

\section{References}

[1] B. Brumitt et al. EasyLiving: Technologies for intelligent environments. In $H U C$, pages 12-29, 2000.

[2] G. Chen and D. Kotz. Supporting adaptive ubiquitous applications with the solar system. Technical Report TR2001-397, Darthmouth College, Hanover, NH, USA, 2001.

[3] N. H. Cohen et al. iQueue: A pervasive data composition framework. In Proceedings of the Third International Conference on Mobile Data Management (MDM), pages 146-153, 8-11 January 2002.

[4] F. Dellaert et al. Recognizing emotion in speech. In Proceedings of Fourth International Conference on Spoken Language, volume 3, pages 1970-1973, 3-6 Oct. 1996.

[5] M. C. Huebscher and J. A. McCann. An adaptive middleware framework for context-aware applications. Personal Ubiquitous Computing, 10(1):12-20, 2005.

[6] C. D. Kidd et al. Mynatt, T. Starner, and W. Newstetter. The Aware Home: A living laboratory for ubiquitous computing research. In Cooperative Buildings, pages 191-198, 1999.

[7] K. V. Laerhoven et al. Towards a wearable inertial sensor network. In Proceedings of the IEE Eurowearable, 2003.

[8] K. Larson and R. Topping. PlaceLab, a House_n + TIAX Initiative. Massachusetts Institute of Technology, Nov. 2003.

[9] E. D. Mynatt et al. Increasing the opportunities for aging in place. In Proceedings on the 2000 conference on Universal Usability, pages 65-71. ACM Press, 2000.

[10] A. Ranganathan et al. Olympus: A high-level programming model for pervasive computing environments. In Third IEEE International Conference on Pervasive Computing and Communications (PerCom), pages 7-16, 8-12 Mar. 2005.

[11] M. Roman et al. A middleware infrastructure for active spaces. Pervasive Computing, IEEE, 1(4):74-83, 2002.

[12] S. Russel and P. Norvig. Artificial Intelligence: A Modern Approach. Prentice Hall, 2nd edition, 2003.

[13] C. Schuldt et al. Recognizing human actions: a local svm approach. In Proceedings of the 17th International Conference on Pattern Recognition, volume 3, pages 32-36, 23-26 Aug. 2004.

[14] V. Stanford. Using pervasive computing to deliver elder care. IEEE Pervasive Computing, 1(1):10-13, 2002.

[15] G. Thomson et al. An approach to dynamic context discovery and composition. Submitted to Ubisys: System Support for Ubiquitous Computing Workshop, UbiComp, 2003. 